\title{
Article
}

\section{Implicit cognitive aggression among young male prisoners: Association with dispositional and current aggression.}

Ireland, Jane Louise and Adams, Christine

Available at http://clok.uclan.ac.uk/12935/

Ireland, Jane Louise ORCID: 0000-0002-5117-5930 and Adams, Christine (2015) Implicit cognitive aggression among young male prisoners: Association with dispositional and current aggression. International Journal of Law and Psychiatry, 41 . pp. 89-94. ISSN 0160-2527

It is advisable to refer to the publisher's version if you intend to cite from the work. dx.doi.org/10.1016/j.jijp.2015.03.012

For more information about UCLan's research in this area go to http://www.uclan.ac.uk/researchgroups/ and search for <name of research Group>.

For information about Research generally at UCLan please go to http://www.uclan.ac.uk/research/

All outputs in CLoK are protected by Intellectual Property Rights law, including Copyright law. Copyright, IPR and Moral Rights for the works on this site are retained by the individual authors and/or other copyright owners. Terms and conditions for use of this material are defined in the policies page.

\section{CLoK}

Central Lancashire online Knowledge www.clok.uclan.ac.uk

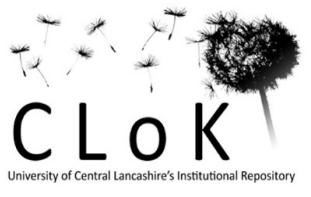


RUNNING HEAD: IMPLICIT AGGRESSION AMONG YOUNG PRISONERS

Implicit cognitive aggression among young male prisoners: Association with dispositional and current aggression.

Jane L. Ireland

Ashworth Research Centre (ARC), Mersey Care NHS Trust and University of Central Lancashire, UK.

Christine Adams

HM Prison Service, UK

KEYWORDS: Prison aggression; Puzzle Test; implicit cognition; DIPC

*Corresponding author; Jane L. Ireland, University of Central Lancashire, Preston, PR1 2HE, UK. E-mail:JLIreland1@uclan.ac.uk; TEL: 01772894471 


\begin{abstract}
The current study explores associations between implicit and explicit aggression in young adult male prisoners, seeking to apply the Reflection-Impulsive Model and indicate parity with elements of the General Aggression Model and Social Cognition. Implicit cognitive aggressive processing is not an area that has been examined among prisoners. Two hundred and sixty two prisoners completed an implicit cognitive aggression measure (PUZZLE Test) and explicit aggression measures, covering current behaviour (DIPC-R) and aggression disposition (AQ). It was predicted that dispositional aggression would be predicted by implicit cognitive aggression, and that implicit cognitive aggression would predict current engagement in aggressive behaviour. It was also predicted that the more impulsive implicit cognitive processing would associate with aggressive behaviour whereas cognitively effortful implicit cognitive processing would not. Implicit aggressive cognitive processing was associated with increased dispositional aggression but not current reports of aggressive behaviour. Impulsive implicit cognitive processing of an aggressive nature predicted increased dispositional aggression whereas the more cognitively effortful implicit cognitive aggression did not. The article concludes by outlining the importance of accounting for implicit cognitive processing among prisoners and the need to separate such processing into facets (i.e. impulsive vs. cognitively effortful). Implications for future research and practice in this novel area of study are indicated.
\end{abstract}


Implicit cognitive aggression among young male prisoners: Association with dispositional and current aggression.

Implicit or 'automatic' processing has been described as a spontaneous process not requiring deliberation, operating in the absence of conscious supervision and intention (Moors \& DeHouwer, 2006). The exact definition has proven challenging to capture (DeHouwer, Teige-Mocigemba, Spruyt \& Moors, 2009), although the most basic will refer to it as a form of cognitive processing that can occur outside of conscious awareness. Definitions of implicit cognitive processing do share commonalities though, brought together by Stacy \& Wiers (2010) who describe it as a subset of associations in memory spontaneously activated under various conditions. These associations can operate without conscious supervision and are thought influenced by experiences. They can impact on emotions and behaviour (Koole, 2009), with the latter including a range of challenging behaviours, such as aggression in general, student and workplace samples (e.g. Todorov \& Bargh, 2002; James et al, 2005; Bluemke, Friedrich \& Zumbach, 2009; Frost, Ko \& James, 2007), and extending to emotional aggression between partners (Ireland \& Birch, 2013).

The impact of implicit cognitive processing on aggression has been of particular interest, with a considerable proportion of aggression thought to occur in the absence of cognitive resources (Bluemke et al, 2009) and not always in line with conscious thought (Banse \& Greenwald, 2007; Perugini \& Banse, 2007). The General Aggression Model (GAM) attempts to capture this by distinguishing between impulsive behaviour and thoughtful action (Anderson \& Bushman, 2002), describing aggression as influenced by the former and demonstrated in the absence of considered deliberation. The GAM was built on pre-existing research from social cognition 
focusing on the role of automatic processing in aggression (Todorov \& Bargh, 2002). The Integrated Model of Information Processing (Huesmann, 1998) is arguably one of the best social cognition models capturing automatic processing, with this model forming an underpinning theoretical element of the GAM. The Integrated Model makes specific reference to automatic cognition as a factor crucial in priming aggressive scripts. The model argues how such cognition leads to an individual accessing an aggressive script (and then enacting it) more rapidly, and then using their resulting aggressive behaviour as a means of justifying the value of the aggressive script. This leads to further reinforcement of the associated aggressive cognitions, including automatic cognitions.

However, the primary model that has captured a role for implicit cognition appears to be the Reflection-Impulsive Model (RIM: Strack \& Deutsch, 2004). This model has not been applied to aggression and yet clearly has valuable components with clear utility to furthering our understanding of aggressive processing at a cognitive level. It describes two systems relevant to implicit cognitive processing, an associative system and a reflective system, both of which are thought to co-exist. The reflective system is consistent more with explicit methods of assessing aggression involving conscious deliberation and appraisal (Hofmann \& Friese, 2008). Such methods include self-report aggression measures which ask directly about aggression frequency and tendency (e.g. 'how often have you shown aggression in the past month?').

The reflective system has featured most heavily in aggression research (e.g. Joormann \& Gotlib, 2010) as opposed to the associative [implicit cognitive] system. The explicit, reflective, system has also featured heavily in aggression therapy where attempts are made to alter beliefs and appraisals by identifying and exploring them 
directly (Ireland, 2011). However, the success of such attempts is questionable regarding their enduring impact on those cognitions which may not be immediately accessible to individuals. It could be argued that prompting change by using the more automatic [implicit cognition] systems may have more success since it would be addressing the more implicit levels of cognition and thus unconscious cognition which has, to date, been neglected in aggression therapy and assessment (Ireland, 2011).

Some researchers have also referred to implicit cognitive processing as a disposition and thus a more stable, trait-related construct. This disposition is thought to play a key role in aggression tendency, particularly with impulsive aggression (Bluemke et al, 2009), a notion supported by both the GAM and the Integrated Model. Using a student sample Bluemke et al (2009) found it correlated with stable trait aggression measures. Bluemke et al (2009) noted a positive correlation between implicit cognitive aggression and overall trait, physical and angry aggression but not in relation to verbal aggression and hostility.

Aggression research to date has, however, failed to address prisoners and has focused on implicit cognitive processing as a homogenous concept. There is considerable heterogeneity in implicit cognitive processing. For example, some can be more impulsive in nature and some more cognitively effortful (DeHouwer, TeigeMocigemba, Spruyt \& Moors, 2009). There is developing consensus that implicit cognitive processing should be considered a multi-factorial concept (Stacy \& Wiers, 2010) which is aptly reflected in the methods used to examine it.

Methods to assess for implicit cognition include the Implicit Association Test (Greenwald, McGhee \& Schwartz, 1998) incorporating response latency; primed Stroop tests (Stewart, Hall, Wilkie \& Birch, 2002) and word association and word 
production tests (Cramer, 1986; Ireland \& Birch, 2013). Word tests have utilised both free-word associations and controlled associations. The former is thought a more impulsive element of implicit cognitive processing and the latter more cognitively effortful (Stacy \& Wiers, 2010). 'Cognitive effortful’ refers here to cognitive processing that still occurs at a largely unconscious level but is less automatic than impulsive cognitive processing. Thus, although it remains a largely unconscious process, therefore distinguishing it from explicit processing, it represents the less automatic element of implicit cognitive processing.

Impulsive implicit processing is of particular interest in forensic populations where impulsive responding is considered a factor underpinning a range of challenging and dissocial behaviours, including aggression. Indeed, aggressive responding is thought a product in part of an absence of cognitive resources and/or of a situation where behavioural control is lacking (i.e. impulsivity) (Bluemke et al, 2009). While aggression research has considered behavioural control in detail by examining areas such as impulsivity using explicit methods of measurement (e.g. Ireland \& Archer, 2004), what has not been considered is the distinction between impulsive implicit cognitive aggressive processing and the more cognitively effortful implicit aggressive processing. It could be logically expected, for example, that it would be the more impulsive implicit cognitive processing likely associated with aggression than that requiring more cognitive effort. Impulsive implicit cognitive processing in this instance would be considered more an extension of explicit impulsive processes (e.g. behaviour) already known to relate to increased aggression (Ireland \& Archer, 2004).

In addition, it could theoretically be expected that increased implicit cognitive aggressive processing would be found more with individuals demonstrating an 
explicit dispositional tendency for aggression (Bluemke et al, 2009) since implicit elements are based on pre-existing content which can be activated implicitly. Those with a tendency towards repeated aggression, as determined by dispositional [trait] aggression, would be expected to have more aggressive memories. On the basis that implicit cognitive processing is based on associative memory (Stacy \& Wiers, 2010) and activates the associative system described by the Reflection-Impulsive Model (RIM: Strack \& Deutsch, 2004), it would be expected that those with a disposition towards aggression have a wealth of aggression memories that become associated when implicit cognitive processing is activated.

As noted, examining the association between implicit cognitive processing and aggression among prisoners represents a neglected field of study. The current study aims to examine such processing with a young adult male prisoner sample, exploring its association with explicit aggressive disposition and current aggressive behaviours, making use of the following models; Reflective-Impulsive Model, Integrated Model of Information Processing and the General Aggression Model. The following core predictions were made: (1) Dispositional aggression would be predicted by implicit cognitive aggression; (2) Implicit cognitive non-aggression will predict decreased dispositional aggression; (3) Implicit cognitive aggression will predict current engagement in aggressive behaviour, (4) The more impulsive implicit cognitive aggression will predict increased aggressive behaviour, with the more cognitively effortful implicit aggression not serving as a predictor.

\section{Method}

\section{Participants}

Three hundred and forty seven young male prisoners were invited to take part from a single establishment. Three hundred and thirty two measures were returned, of 
which 262 were completed. This represented a 75.5 percent completion rate. The sample was a general prisoner sample; thus prisoners were not selected from this sample due to increased levels of aggressive tendency.

The mean age of participants was 19.4 years (SD0.8: age range 18 to 21). Eighty seven percent classed their ethnicity as white, three percent as Asian, four percent black, three percent mixed race, with one percent not indicating ethnicity. Of those sentenced, the majority (38 percent) were serving for an acquisitive offence (e.g. theft, burglary) and 33 percent for a violent offence (e.g. wounding or assault). Eight percent were serving for drug offences, 19 percent for other offences (e.g. arson, motoring offences), with the remaining two percent not stating what they were detained for. The mean sentence length was 5.4 years (SD 2.8) and mean total length of time spent in prison 3.6 years (SD 1.6). Sixteen percent were not sentenced as they remained on remand awaiting sentencing/outcome.

\section{Measures}

All participants completed the following measures.

Puzzle Test (Ireland \& Birch, 2013).The Puzzle Test is a variant of a word association test which incorporates cognitively effortful (less automatic) and uncontrolled (impulsive) implicit cognitive processes using two core methods: word identification and word replacement. Such word association tests are considered capable of identifying implicit conceptual memory (e.g. Zeelenberg, Shiffrin \& Raaijmakers, 1999). The word identification element of the Puzzle Test is a variant of free-word association tests where instead of generating the first word that comes to mind following a cue, participants are asked to identify the first word that they can identify from two pre-prepared word searches. It is designed to measure more impulsive cognitive responding. The word replacement element requires the insertion 
of a number of words into two crossword-style puzzles where there are cues in place (e.g. occasional letters: see Appendix 1). The word replacement element of the test requires more cognitive effort to complete. It is thus less automatic in processing and is focused more on cognitively effortful implicit associations. The Puzzle Test focuses on the implicit cognitive tendency to identify aggression and also nonaggression.

\section{Direct and Indirect Prisoner Behaviour Checklist - revised (DIPC-R:}

Ireland 2005). The DIPC-R aims to identify current instances of aggression that have occurred between prisoners in the past week. It outlines 111 sets of behaviours which assess two types of aggression; direct (e.g. physical, psychological, verbal) and indirect (e.g. gossiping, spreading rumours, practical jokes), covering both victimisation and perpetration. Examples of items include 'I have been kicked or hit by another prisoner', 'I have been gossiped about', 'I have physically threatened another prisoner with violence' and 'I have spread rumours about someone'. It includes a range of other items (e.g. involvement in drugs, filler items etc) that were not utilised in the current study. The DIPC (Ireland \& Ireland, 2008) has been validated on men, women, adults, and adolescents within prisons. As a behavioural measure, it has been used extensively within prisons (e.g., Archer \& Southall, 2009; Lawrence \& Welfare, 2008). It is included here as an explicit measure of aggression, namely one that asks directly about aggressive behaviours.

Aggression Questionnaire (AQ: Buss \& Perry, 1992). The AQ is a 28 item measure assessing dispositional (trait) aggression. Items are scored on a Likert scale from 1 (extremely unlike me) to 5 (extremely like me). It comprises four subscales: physical aggression, verbal aggression, anger, and hostility. Examples of items are 'I have become so mad I have broken things' (physical), 'I am suspicious of overly 
friendly strangers' (hostile), 'I have trouble controlling my temper' (anger) and 'I often find myself disagreeing with people' (verbal). It represents a further explicit measure of aggression since it enquires directly about aggressive tendencies, commonly referred to as dispositions.

\section{Procedure}

Ethical approval for the study was gained from the University of Central Lancashire Ethics Committee and from the prison via local approval procedures. All prisoners at the time of data collection were invited to take part. Participation was voluntary. Questionnaires were distributed during a lunchtime period when prisoners were on 'lock-up’ (i.e. all within their cells). All questionnaires were preceded by an information sheet. A blank envelope was provided for prisoners to place their completed (or uncompleted) questionnaire in. Questionnaires were collected approximately one hour later, at the end of the lunch period.

\section{Results}

Findings in relation to implicit cognitive aggression and explicit aggression (i.e. behavioural and aggressive disposition) are presented first, before proceeding onto an examination of the associations and predictions between variables. This will be completed by examining overall implicit cognitive processing first, before exploring the facets of impulsive and cognitively effortful implicit responding. Table 1 presents the means across the sample regarding implicit cognitive tendencies. Table 2 presents proportions of aggressive behaviours reported (DIPC-R), including presentation of the explicit dispositional aggression measure (AQ).

$<$ Insert Table 1 here $>$ 
There was no difference between the total number of aggressive words detected and the number of non-aggressive words $(t=.52 n s)$ on the implicit cognitive measure.

$<$ Insert Table 2 here $>$

There were no differences between direct and indirect aggression, either for perpetration or victimisation, with approximately half the sample reporting engagement in the perpetration of aggression towards others and half reporting victimisation. One third of prisoners reported both perpetration and victimisation items. Across dispositional aggression, the highest scores were indicated on physical aggression, followed by hostility and anger.

\section{Association between overall implicit cognitive processing and explicit dispositional aggression}

Regressions were utilised to determine if implicit cognitive aggression and implicit cognitive non-aggression could be predicted by dispositional aggression, as determined by the Aggression Questionnaire (AQ). Four regressions were completed: two addressing the AQ subscales independently (i.e. physical, verbal, anger, hostility) in relation to the total number of implicit cognition non-aggressive and implicit cognition aggressive words, and two repeated but in relation to total AQ scores. All regressions were completed using the Enter method.

Regarding the number of aggressive words identified on the implicit cognitive test [the Puzzle test], the regression regarding overall AQ scores was significant $(F=$ 4.66, $\operatorname{df}(1,260), p<.03)$ with increased dispositional aggression predictive of increased implicit cognitive aggression $(\beta=.03, T=2.16, p<.03)$. Across AQ subscales, the regression was significant overall $(F=2.60$, df $(4,257), p<.04)$ but the 
only significant predictor was hostility, with decreased levels of dispositional hostility associated with increased levels of aggressive words being identified implicitly ( $\beta=$ $.12, T=-1.65, p<.05)$.

Regarding the number of non-aggressive words identified on the implicit cognitive test, total AQ scores represented a significant predictor $(F=3.74$, df $(1$, $260), p<.05$ ), with decreased dispositional aggression predictive of increased nonaggression on the implicit cognition test $(\beta=-.12, T=-.1 .93, p<.05)$. The regression across AQ subscales was not significant $(F=2.03 \mathrm{~ns}$ ) indicating that it was the composition of aggression across subscales serving as a predictor.

\section{Association between overall implicit cognitive processing and explicit current aggression}

Regressions were then completed to determine if implicit cognitive aggression and implicit cognitive non-aggression could be predictors of current behavioural difficulties, namely aggression as measured via the DIPC-R. Two regressions were completed, examining whether aggressive implicit cognitive processing and nonaggressive implicit cognitive processing predicted the DIPC-R totals for perpetration and victimisation. Four further regressions examined if implicit cognitive processing predicted indirect perpetration, direct perpetration, indirect victimisation and direct victimisation. All regressions were completed using the Enter method.

Implicit cognitive processing, either aggressive or non-aggressive, did not predict total perpetration scores $(F=.27 \mathrm{~ns})$ or total victimisation scores. It also did not predict total direct perpetration $(F=.68 \mathrm{~ns})$, total indirect perpetration $(F=.68 \mathrm{~ns})$, direct victimisation $(F=.61 \mathrm{~ns})$ or indirect victimisation $(F=.90 \mathrm{~ns})$. 


\section{Association between impulsive and cognitively effortful implicit cognitive processing and explicit dispositional aggression}

Implicit cognitive processing was examined in more detail by considering individually those elements of the Puzzle Test designed to assess impulsive implicit cognitive processing (i.e. word identification) and those examining more cognitively effortful implicit processing (i.e. word generation). Regressions were completed as for overall implicit cognitive testing.

Considering impulsive implicit cognitive aggression, the regression regarding overall AQ scores was significant $(F=5.02$, df $(1,260), p<.02)$ with increased trait aggression predictive of an increased tendency for impulsive implicit cognitive aggression $(\beta=.13, T=2.24, p<.02)$. Across AQ subscales, although the overall regression was significant $(F=2.62, \mathrm{df}(4,257), p<.03)$, there were no significant individual predictors (all $\beta$ 's $\leq 1.56$ ). A similar pattern was found in relation to impulsive non-aggressive implicit words: the regression regarding overall AQ scores was significant $(F=5.20$, df $(1,260), p<.02)$ with decreased trait aggression predictive of an increased tendency to identify impulsive implicit non-aggression $(\beta=$ $-.14, T=-2.28, p<.02)$. Across AQ subscales, the regression was not significant $(F=$ 2.08ns).

Considering more cognitively effortful aggressive implicit processing (i.e. word generation), the regression regarding overall AQ scores was not significant $(F=$ 2.54ns), and nor was it for AQ subscales ( $F=2.09 \mathrm{~ns})$. This also held for the generation of cognitively effortful non-aggressive implicit words, both for overall trait $\operatorname{aggression}(F=.83 \mathrm{~ns})$ and the subscales of trait aggression $(F=1.29 \mathrm{~ns})$. 


\section{Association between impulsive and cognitively effortful implicit processing and explicit current aggression}

Regressions were then completed to determine if impulsive or cognitively effortful implicit cognition could predict current behavioural difficulties, namely aggression as measured on the DIPC-R. Implicit cognitive processing, neither aggressive nor non-aggressive, did not predict total perpetration scores ( $F=.45 \mathrm{~ns})$. Regarding total victimisation scores the individual regression was significant for impulsive implicit cognitive aggression. Thus, this was repeated entering just impulsive implicit cognitive aggression. The resulting regression was significant $(F=$ 4.32, df (1,260), $p<.03)$, indicating that increased impulsive implicit cognitive aggression was a predictor for decreased reports of being victimised $(\beta=-.25, T=-$ $2.08, p<.04)$.

Impulsive implicit cognitive processing did not predict total direct perpetration $(F=1.02 \mathrm{~ns})$, total indirect perpetration $(F=.49 \mathrm{~ns})$ or direct victimisation $(F=$ 1.61ns). For indirect victimisation the individual regression was significant just for impulsive implicit cognitive aggression. Thus, this was repeated entering just impulsive implicit cognitive aggression as a predictor. The resulting regression was significant $(F=4.50$, df $(1,260), p<.03)$, indicating that increased impulsive implicit cognitive aggression was a predictor for decreased reports of being victimised indirectly $(\beta=-.11, T=-2.12, p<.04)$.

Examining more cognitively effortful implicit processing; regressions were completed as for impulsive processing. No regressions were significant (all $F$ 's $\leq=$ $.94)$. 


\section{Discussion}

An association between increased implicit cognitive aggression and increased dispositional aggression among prisoners was indicated. Dispositional aggression was predicted by impulsive implicit cognitive processing but not by the more cognitively effortful (i.e. less automatic) implicit processing. The association between implicit cognitive aggression and dispositional (explicit) aggression supports previous research with other samples using a range of other methods (e.g. Todorov \& Bargh, 2002; James et al, 2005; Frost, Ko \& James, 2007; Bluemke et al, 2009; Ireland \& Birch, 2013).

It also lends support to the argument that implicit cognition is based on associative memories for aggression; thus, those with dispositional tendencies towards aggression are expected to have more memories of this kind that become accessibly at a largely unconscious (i.e. implicit) level, leading to higher levels of implicit cognitive aggression. This is speculative but is argued to represent one means by which the reflective system is activated (Stacy \& Wiers, 2010). The current study suggests such an argument could be extended to prisoners, supporting the predictions that dispositional aggression would be predicted by implicit cognitive aggression, with reduced aggression disposition predicted by implicit cognitive non-aggression.

The findings clearly suggest a role for automatic processing in aggression (Anderson \& Bushman, 2002; Todorov \& Bargh, 2002) which is linked to habitual aggression due to its dispositional component. It supports both the General Aggression Model (GAM: Anderson \& Bushman, 2002) and the Integrated Model of Information Processing which argues for a significant role for automatic (implicit) cognition in aggression (Huesmann, 1998). The findings further support the Reflection-Impulsive Model (RIM: Strack \& Deutsch, 2004), suggesting co-existence 
between the associative [implicit cognition] system and the reflective [explicit] system, with the latter characterised here by dispositional aggression. A role for both explicit (Hofmann \& Friese, 2008; Joormann \& Gotlib, 2010) and implicit systems are thus indicated and are occurring concurrently.

The explicit [reflective] system is not extending to more dynamic instances of aggression (i.e. current aggression), suggesting that the Reflective-Impulsive system outlined by the RIM may be more dispositional in nature. The current findings suggest that the association is clearly focused on dispositional aggression as opposed to more current aggressive behaviours where the prediction that implicit cognitive aggression would predict current [i.e. explicit] aggression was unsupported.

The relationship between implicit cognitive and dispositional aggression was focused on the collection of subscales of aggression disposition, namely overall trait aggression. It did not extend to the subscales of anger, verbal or physical aggression. Thus the prediction that aggression disposition would be predicted by implicit cognitive aggression was supported only in relation to overall dispositional aggression and not when exploring the individual subscales of trait aggression. This finding also held in relation to dispositional aggression and implicit cognitive non-aggression. It appears the combination of dispositional aggression types that represents the significant factor. Indeed, only one subscale, hostility, appeared associated with increased implicit cognitive processing, but in an unexpected direction; decreased hostility was associated with increased aggressive words. However, this was not a predicted finding and the size of the predictor was low $(\mathrm{T}=1.65)$. Arguably this level should be rejected since it was not predicted. It adds to the suggestion, however, that it is the composition of overall trait aggression that is contributing to the association 
with increased implicit aggressive processing and that the subscales of trait aggression may be unreliable predictors.

The current study also noted the importance of accounting for the heterogeneity of implicit cognitive processing by examining both impulsive and more cognitively effortful components (DeHouwer et al, 2009; Stacy \& Wiers, 2010). As noted, impulsive implicit cognitive processing was associated with dispositional aggression and not the more cognitively effortful implicit processing. This supported the prediction made and was consistent with the General Aggression Model which outlines a role for more impulsive elements promoting aggression (Anderson \& Bushman, 2002). Clearly, the more impulsive implicit cognitive processing is most closely aligned with the automatic element of the Reflection-Impulsive Model (RIM: Strack \& Deutsch, 2004) since the absence of deliberate cognitive processing is arguably greater. More cognitively effortful implicit processing arguably becomes more closely linked with the reflective (explicit) elements of such models due to the increased cognitive effort and thus less automatic nature.

This suggests viewing implicit cognitive processing along a continuum ranging from automatic to reflective is more helpful and realistic. Current research does not capture implicit cognitive processing along a continuum, focusing instead on describing systems that co-exist but are parallel (i.e. either reflective or automatic). Viewing implicit cognitive processing along a continuum may have more value and allow for more detailed examination of individual variations and how these link to aggression.

Regarding current involvement in aggression, an unexpected finding was noted in relation to reports of victimisation. Increased levels of victimisation were predicted by decreased levels of impulsive implicit cognitive processing, suggesting that 
prisoners with increased levels of impulsive implicit cognitive aggression were less likely to report victimisation by other prisoners. This also held for indirect victimisation. In reflection of the finding that impulsive implicit cognitive aggression predicted higher levels of dispositional aggression, this could simply suggest that victims of aggression in prison are characterised by increased levels of dispositional aggression, which would be consistent with the finding that one third of the sample were engaging in both perpetration and victimisation. The concept of a mutual perpetrator-victim group in prisons is well recognised (Ireland \& Archer, 2004; Ireland, 2005) and it could be argued that the current findings are reflective of a tendency for prison victimisation to be associated also with perpetration. However, it does suggest a relationship between elements of increased levels of victimisation (not increased levels of perpetration) and implicit cognitive aggression which is perhaps worthy of further study, highlighting further how the interplay between implicit cognitive processing and its facets may not be straightforward within a forensic (prisoner) sample. The importance of separating the facets of implicit cognitive processing is certainly indicated by the current findings.

The current study is not without its limitations. The study relies on self-report measures with limitations including honesty in reporting, understanding and ability, and a disbelief in their responses remaining anonymous and secure. Furthermore, the current study did not separate implicit cognitive process from any emotional response (e.g. anger) that could have been triggered by the measures. Emotions are undoubtedly a key element that can drive behavioural responses and cognitions linked to increased levels of aggression (e.g. Ireland, 2011). It is certainly possible that exposure to an aggressive word (such as that indicated on the Puzzle Test), could have triggered a linked emotional response with this emotional response then dictating the 
reaction to the measure as opposed to the implicit cognitive processes. Implicit cognitive and implicit emotional systems are closely aligned and what is being indicated here is the potential for an implicit emotional system to have impacted.

Limitations are unavoidable in a detained sample, however, where restrictions on methodology are further dictated by organisational requirements and expectations. Nevertheless, the study accepts that there are limitations and offers the current findings as initial results designed to prompt further research and evaluation of implicit cognitive processing in prison samples. There is also convergence in findings with previous published research utilising other samples, despite variations in method, which supports some generalisation of the findings.

Future research could explore the use of different methods for assessing implicit cognitive processing, perhaps by incorporating response latency and also incorporating more objective data on aggression and prisoner characteristics. There are significant challenges in conducting more experimental research within the constraints of secure settings, and a significantly reduced sample size likely. Nevertheless, additional variables could be incorporated into future studies. This includes the role of implicit emotion (Joormann \& Gotlib, 2010) which could serve to amplify negative behavioural responding by promoting explicit cognition, such as rumination, known to escalate the risk for aggression (Ireland, 2011). Indeed, the area of implicit (unconscious, automatic) emotion and its regulation is not one that has been addressed to date in forensic populations and yet may clearly relate to implicit cognitive processing and treatment outcome.

Examining differences within implicit cognitive processing would also have value: the importance of capturing the facets of such processing has already been indicated, but the role of efficiency in implicit cognitive processing could further be 
examined. This represents the cognitive capacity of an individual and their ability to control or implicitly regulate their implicit cognitive processing. This may be crucial for intervention and thus worthy of further investigation with prisoners.

Overall, the current study illustrates a role for implicit aggressive cognitive processing and dispositional aggression, indicating associations between them. The more cognitively effortful implicit processing was not a contributing component to dispositional aggression. These findings are important owing to the lack of research in this area with prisoners and an apparent omission within risk assessments and therapy to capture the implicit cognitive processing system (Ireland, 2011). To date, focus regarding cognition has centred on the reflective (explicit) element of the RIM as opposed to the automatic component. This omission has led to a focus on treating and understanding the explicit aggression system and not the implicit when the latter is so clearly associated with an amalgamation of dispositional aggression.

The findings suggest that the assessment and treatment of aggression in forensic (prisoner) samples should attend to the implicit system of cognitive processing, particularly when trying to formulate and treat more habitual, dispositional, aggression. These findings offer useful preliminary data on the value of the implicit cognitive system in understanding aggression disposition in high risk groups such as prisoners, and a need to examine such systems in more detail.

\section{References}

Anderson, C.A., \&Bushman, B.J. (2002). Human Aggression. Annual Review of Psychology, 53, 27 - 51. Doi: 10.1146/annurev.psych.53.100901.135231. Archer, J., \& Southall, N. (2009). Does cost-benefit analysis or self control predict 
involvement in bullying behavior by male prisoners? Aggressive Behavior, 35, 31-40. doi:10.1002/ab.20283.

Banse, R., \&Greenwald, A.G. (2007). Personality and implicit social cognition research: Past, present and future. European Journal of Personality, 21, 371 382. doi: 10.1002/per.638.

Bluemke, M., Friedrich, M.,\& Zumbach, J (2009). The influence of violent and nonviolent computer games on implicit measures of aggressiveness. Aggressive Behavior, 35, 1 - 13. doi: 10.1002/ab.20329.

Buss, A.H., \& Perry, M. (1992). The Aggression Questionnaire. Journal of Personality and Social Psychology, 63, 3,452 - 459.

Cramer, P. (1968). Word Association. Academic, Saint Louis, MO.

De Houwer, J.,Teige-Mocigemba, S., Spruyt, A.,\& Moors, A. (2009). Implicit measures: A normative analysis and review. Psychological Bulletin, 135, 347 - 68. doi: 10.1037/a0014211.

Frost, B. C., Ko, C. E., \& James, L. R. (2007). Implicit and explicit personality: A test of a channeling hypothesis for aggressive behavior. Journal of Applied Psychology, 92, 1299 - 1319. doi: 10.1037/0021-9010.92.5.1299.

Greenwald, A.G., McGhee, D.E.,\& Schwartz, J.L.K. (1998). Measuring individual differences in implicit cognition: the Implicit Association Test. Journal of Personality \& Social Psychology, 74: 1464 - 80.doi:10.1037/00223514.74.6.1464.

Hofmann, W.,\& Friese, M. (2008). Impulses got the better of me: Alcohol moderates the influence of implicit attitudes towards food cues on eating behaviour. Journal of Abnormal Behavior, 117, 420 - 427. doi: 10.1037/0021843X.117.2.420. 
Huesmann, L. R. (1998). The role of social information processing and cognitive schema in the acquisition and maintenance of habitual aggressive behavior. In R. G. Geen.,\& E. Donnerstein (Eds.), Human aggression: Theories, research, and implications for social policy. London, UK: Academic Press Ltd.

Ireland, J.L., \& Archer, J. (2004). Association between measures of aggression and bullying among juvenile and young offenders. Aggressive Behaviour, 30, 2942.doi: 10.1002/ab.20007.

Ireland, J. L. (Ed.). (2005).Bullying in Prison: Innovations in research and theory. Willan Publishing, UK: Devon.

Ireland, J. L. (2011). Violence assessment and intervention. In G. Davey (Ed.), Applied Psychology (pp. 291 - 316). West Sussex, UK: BPS WileyBlackwell.

Ireland, J.L.,\& Birch, P. (2013). Emotionally abusive behaviour in young couples: Exploring a role for implicit aggression. Journal of Interpersonal Violence, 210, 375 - 380. http://dx.doi.org/10.1891/0886-6708.VV-D-12-00021.

Ireland, J. L., \& Ireland, C. A. (2008). Intra-group aggression among prisoners: Bullying intensity and exploration of victim-perpetrator mutuality. Aggressive Behavior, 34, 76-87. doi:10.1002/ab.20213.

James, L. R., McIntyre, M. D., Glisson, C. A., Green, P. D., Patton, T. W., LeBreton, J. M., Frost, B. C., Russell, S. M., Sablynski, C. J., Mitchell, T. R., \& Williams, L. J. (2005). A conditional reasoning measure for aggression, Organizational Research Methods, 8, 69-99. doi: 10.1177/1094428104272182 Joormann, J.,\& Gotlib, I.H. (2010). Emotion regulation in depression: Relation to cognitive inhibition. Cognition \& Emotion, 24, 913 - 939. doi: 10.1080/02699930903407948. 
Koole, S.L. (2009). The psychology of emotion regulation: An integrative review. Cognition \& Emotion, 23, 4 - 41. doi:10.1080/02699930802619031.

Lawrence, S., \& Welfare, H. (2008). The effects of the introduction of the nosmoking policy at HMYOI Warren Hill on bullying behaviour. International Journal of Prisoner Health, 4, 134-145. doi:10.1080/17449200802264662.

Moors, A.,\& DeHouwer, J. (2006). Automaticity: A theoretical and conceptual analysis. Psychological Bulletin, 132, 297 - 232. doi: 10.1037/00332909.132.2.297.

Perugini, M.,\&Banse, R. (2007). Personality, implicit self-concept andautomaticity. European Journal of Personality, 21: 257 - 261.Doi: 10.1002/per.637. Stacy, AW.,\& Wiers, RW. (2010). Implicit cognition and addiction: A tool for explaining paradoxical behaviour. Annual Review Clinical Psychology, 6: 551 - 575. doi: 10.1146/annurev.clinpsy.121208.131444.

Strack, F.,\& Deutsch, R. (2004). Reflective and impulsive determinants of social behaviour. Personality Social Psychology Review, 8, 220 - 247.

Stewart, S.H., Hall, E., Wilkie, H., \& Birch, C.D. (2002). Affective priming of alcohol schema in coping and enhancement motivated drinkers. Cognitive Behavioural Therapy, 31: $68-80$.

Todorov, A.,\& Bargh, J.A. (2002). Automatic sources of aggression. Aggressive \& Violent Behavior, 7: 53 - 68. http://dx.doi.org/10.1016/S1359-789(00)00036$\underline{7}$.

Zeelenberg, R., Shiffrin, R.M., \& Raaijmakers, J.G.W. (1999). Priming in a free association task as a function of association directionality, Memory \& Cognition, 27:956 - 961. doi.10.3758/BF03201226. 
Appendix 1

Example of one of two tests from the Puzzle Test designed to assess cognitively

effortful implicit aggressive processing (@Taken from Ireland \& Birch, 2013).

Your aim below is to complete as many of the white boxes as you can using a word that fits. It does not matter what word you use but it must be a word (not a name or place) and include the letters already in the grid. You must not write in the shaded areas. One word has already been completed to help start you off. Just try and fill in as many as you can.

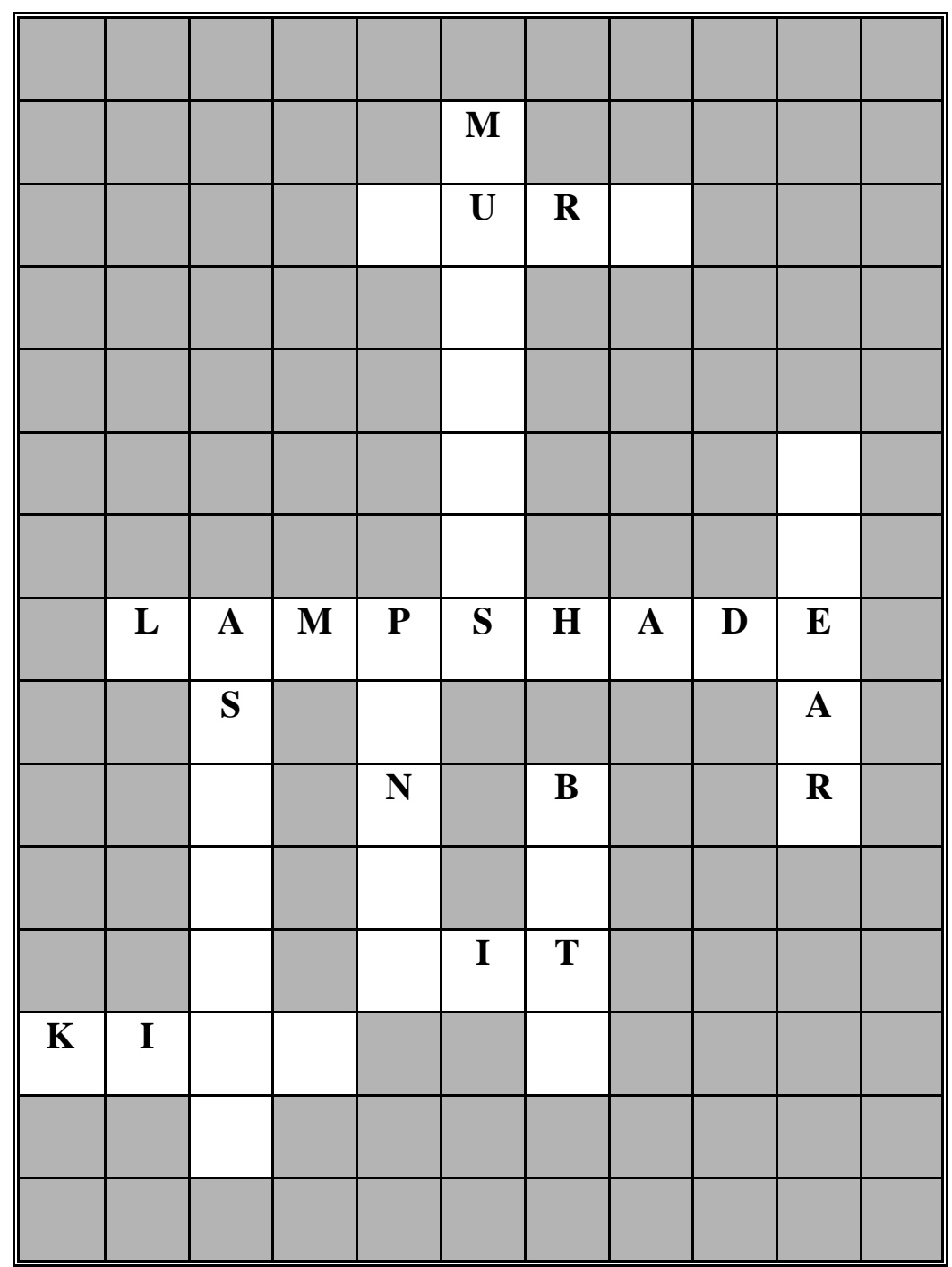


Table 1

Means across implicit test (Puzzle Test: $\mathrm{n}=262$ )

Implicit Measure - Puzzle Test

Impulsive aggressive

Impulsive non-aggressive

Cognitively effortful aggressive

Cognitively effortful non-aggressive

Total implicit: aggressive

Total implicit: non-aggressive

\section{Mean (SD/n)}

$6.1(2.6 / 262)$

$7.6(3.4 / 262)$

$6.1(3.4 / 262)$

$4.5(3.1 / 262)$

$12.3(5.2 / 262)$

$12.1(5.5 / 262)$ 
Table 2

Explicit assessment of behaviour: Proportion of aggression and challenging behaviours on the DIPC-R and means on the Aggression Questionnaire

\begin{tabular}{ll}
\hline Explicit Measure - DIPC-R & \% (n = 262) \\
\hline Direct aggression* & 34 \\
Indirect aggression* & 39 \\
Total aggression* & 47 \\
Direct victimisation & 34 \\
Indirect aggression & 41 \\
Total victimisation & 50 \\
\hline Explicit Measure - Aggression Questionnaire & Mean (SD), n = 262) \\
\hline Physical & $28.6(8.3)$ \\
Verbal & $16.6(4.4)$ \\
Anger & $20.2(6.6)$ \\
Hostility & $21.9(6.9)$ \\
Total & $87.4(21.7)$ \\
\hline *perpetration of aggression & \\
\hline
\end{tabular}

